\title{
Repetitive transcranial magnetic stimulation as a prophylactic treatment in migraine
}

\author{
Azza B. Hammad, Rasha E. Elsharkawy and Ghada S. Abdel Azim* (D)
}

\begin{abstract}
Background: Clinical applications of transcranial magnetic stimulation (TMS) have shown promising results in the treatment of headache disorders, with migraine being one of the most encountered.

Objective: To assess the role of low-frequency repetitive transcranial magnetic stimulation as a preventive treatment of migraine (with and without aura) and correlate the results with the serum level of the inflammatory biomarker (neurokinin A).

Methods: Forty patients, with age ranging from 15 to 55 years, diagnosed with migraine (30 migraine without aura and 10 with aura) and 20 apparently healthy individuals, who were age and sex matched with the patient group, were included in this study.

A low-frequency $(1 \mathrm{~Hz}$ ) rTMS protocol was applied for all patients for five consecutive days interictally. Assessment of pain intensity using visual analogue scale and frequency and duration of attacks as well as number of pills taken by patients as an abortive treatment according to the Basic Diagnostic Headache Diary for 4 weeks before and 4 weeks after TMS sessions was done. In addition, the Migraine Disability Assessment scale (MIDAS) was applied to assess the severity and degree of disability caused by migraine.

Measurement of neurokinin A serum level was done by using ELISA for all patients before and after TMS and for control group once.
\end{abstract}

Results: There was a significant reduction in pain intensity, frequency and duration of migraine attacks, migraine disability scores, and number of pills taken as abortive treatment for attacks after rTMS $(P<0.001)$. Also, serum level of neurokinin A in the patients was significantly reduced after rTMS $(P<0.001)$.

Conclusion: Low-frequency rTMS is an effective prophylactic treatment for migraine with and without aura.

Keywords: Migraine, Transcranial magnetic stimulation, Neurokinin A

\section{Introduction}

Migraine is a neurological disorder affecting about $15 \%$ of the population, with a genetic, environmental, and hormonal basis. This pathology causes continuous and severe episodes of headache, and in some cases, it also causes nausea, vomiting, and photophobia [1].

Despite the high prevalence of this headache, many of the genetic causes and physiopathological mechanisms

\footnotetext{
* Correspondence: ghada_saed2006@yahoo.com

Neurology Department, Faculty of Medicine - For Girls, Al Azhar University, Cairo, Egypt

\section{Springer Open}

are still unknown, which makes it harder to find efficient treatments [2].

Studies have shown the pathophysiology of migraines to involve complex biochemical changes that lead to pain. One underlying mechanism is cortical spreading depression (CSD) of the neocortex and hippocampus, which then activates the trigeminal nucleus caudalis, causing vasodilation of blood vessels and release of calcitonin gene-related peptide (CGRP), substance $\mathrm{P}$, and vasoactive intestinal polypeptide, ultimately leading to meningeal irritation and pain. Other changes include increased 5-hydroxytryptamine (5-HT) and nitric oxide $(\mathrm{NO})$ levels, leading to enhanced central sensitization and pain [3]. 
Pharmacologic therapies of migraine such as simple analgesics and non-steroidal anti-inflammatory drugs remain the first-line treatment. However, many individuals continue to have headaches refractory to various prophylactic and/or abortive therapies, while others are at risk of medication overuse headaches [4].

There are a number of non-pharmacologic, noninvasive neurostimulation techniques being researched, which include transcranial magnetic stimulation (TMS), transcranial direct current stimulation (tDCS), transcranial alternating current stimulation (tACS), and transcutaneous electrical nerve stimulation (TENS) [5].

Among non-pharmacologic therapies, repetitive transcranial magnetic stimulation (rTMS), a non-invasive neuromodulation technique involving repeated series of dynamic magnetic impulses, has been studied as a preventive migraine treatment [6].

Although the exact mechanisms through which rTMS works are not completely known, it is considered to be multifactorial. The applied electric charges induced by the magnetic field cause various neurochemical changes such as increased dopamine levels in the hippocampus, reduction in raclopride $\mathrm{C} 11$ binding in the caudate nucleus, fluctuations in glutamate/glutamine levels at the site of rTMS, and increased plasma $\beta$ endorphin levels [4].

Furthermore, rTMS has been shown to induce weak electrical currents, causing depolarization of neurons and inhibition of cortical spreading depression (CSD), which ultimately terminates the aura and reduces the duration or severity of migraine [7]. Additionally, rTMS at the motor cortex is thought to reduce pain by altering the motor cortex projections to the medial thalamus and anterior cingulate/orbitofrontal cortices, thereby modulating pain [4].

Previous findings on rTMS as a therapy for migraine have been inconsistent partly due to no standardized protocol regarding area of stimulation, Frequency, amount of pulses, intensity, and sessions needed to achieve reliable outcomes. Low-frequency rTMS $(1-3 \mathrm{~Hz})$ and high-frequency rTMS $(10-20 \mathrm{~Hz})$ over the dorsolateral prefrontal cortex consistently have not demonstrated effective outcomes and have not performed better than sham treatments. However, there have been promising results following repetitive transcranial magnetic stimulation of the motor cortex for several pain conditions, including migraine [8].

Neurovascular inflammation is characterized by the release of potent vasoactive neuropeptides, predominantly calcitonin gene-related peptide, substance $\mathrm{P}$, and neurokinin A from activated peripheral nociceptive sensory nerve terminals. These peptides lead to a cascade of inflammatory tissue responses including arteriolar vasodilatation, plasma protein extravasation, and degranulation of mast cells in their peripheral target tissue. Neurovascular inflammatory processes have long been implicated as a possible mechanism in the pathophysiology of migraine [9].
In this study, we evaluate the role of low-frequency rTMS at $1 \mathrm{~Hz}$ over the vertex as a preventive treatment of migraine (with and without aura) and correlate the results with serum level of the inflammatory biomarker (neurokinin A).

\section{Methods}

This study included 40 patients who were diagnosed with migraine (six of them are males and 34 are females). They were divided into two groups: group A, 30 patients with migraine without aura, and group B, 10 patients with aura; all patients were selected from outpatient clinic of the Neurology Department of Al Zahraa University Hospital. The patients' age ranged from 15 to 55 years, and they fulfilled the criteria of migraine with aura and migraine without aura based on the International Classification of Headache Disorders 3rd edition [10]. Patients on prophylactic treatment for migraine and those with severe types of migraine (e.g., familial hemiplegic migraine or migraine with brain stem aura) and epileptic patients were excluded from the study. In addition, patients with other types of headache, other neurological diseases, or psychiatric diseases and patients with pacemaker or metallic implant were also excluded.

Also, 20 apparently healthy subjects, who were age and gender matched with the patient group, were included as a control group for serum levels of neurokinin A only. The Ethical Committee of the Faculty of Medicine of Al Azhar University approved the study. In addition, an informed consent was signed by every patient for participation in the study.

Patients had full clinical general and neurological examination. Every patient had a headache diary to record the frequency and duration of attacks as well as number of pills taken as an abortive treatment during 4 weeks before and 4 weeks after TMS sessions according to the Basic Diagnostic Headache Diary (BDHD) [11].

Pain intensity was assessed by using visual analogue scale (on scale from 0 to 10 ), and the severity and degree of disability of migraine were assessed by using the Migraine Disability Assessment scale (MIDAS) 4 weeks before and 4 weeks after TMS sessions.

Interictally, rTMS treatment was performed on five consecutive days using Neuro-MS/D Therapeutic device (Neurosoft Ltd., Ivanovo, Russia). A repetitive magnetic stimulator was used to apply two trains of 500 pulses separated by a 1min interval between the trains at a frequency of $1 \mathrm{~Hz}$ over vertex (Cz, 10-20 EEG System). The center of figure-of-eight coil magnetic stimulator was applied on the vertex.

The stimulation intensity was set to visual motor threshold of the dominant hand, an intensity that corresponds approximately to the resting motor threshold.

\section{Assessment of visual motor threshold}

We assessed the individual motor threshold of the first dorsal interosseous muscle of the right hand for each 
subject by visual inspection. This was performed by putting the probe (figure of eight) on the patient scalp over the region of right hand motor cortex and starting stimulation with single pulses with different intensities. We determined the stimulator intensity that produced a visually detectable minimal muscle contraction in the target muscle in at least 5 out of $10(50 \%)$ trials.

Low-frequency TMS was used based on the rationale that cortical hyperexcitability is proposed to be the putative basis for the pathophysiological disturbances in migraine.

Neurokinin A serum level was estimated for the control group and during interictal period immediately before and after TMS treatment for the patient group using the RayBio (RayBiotech Life, Peachtree Corners, GA, USA) Neurokinin A Enzyme Immunoassay (EIA) Kit which is an in vitro quantitative assay for detecting neurokinin A peptide based on the competitive enzyme immunoassay principle.

\section{Study design}

This is a clinical controlled prospective study.

\section{Statistical analysis}

Data were analyzed using the Statistical Program for Social Science (SPSS) version 21.0. Qualitative data were presented as numbers and percentages and compared between groups using chi-square test while quantitative data were presented in the form of mean, standard deviation (SD), range, median, and interquartile range (IQR) according to their distribution. The comparison between two independent groups regarding quantitative data with parametric distribution was done by using independent $t$ test while comparison of quantitative data with non-parametric distribution was done by using the Mann-Whitney test. Also, the comparison between two independent groups regarding quantitative data with parametric distribution was done by using paired $t$ test while comparison of quantitative data with nonparametric distribution was done by using Wilcoxon's rank test. Spearman's correlation coefficient test was used to assess the correlation between two quantitative parameters in the same group. The confidence interval was set to $95 \%$ and margin of error accepted $5 \%$. So, the $P$ value was considered significant at the level of $<0.05$.

\section{Results}

\section{Characteristics of the study population}

The study included 40 migraine patients (34 females $(85.0 \%)$ and six males (15.0\%)), with a mean age of 30.53 \pm 8.47 years. The control group included 20 subjects (13 females $(65.0 \%)$ and seven males $(35.0 \%)$ ) with a mean age of $29.75 \pm 4.94$ years. Thirty Patients with migraine without aura (group A) (25 females (83.3\%) and five males $(16.7 \%))$ had a mean age of $31.53 \pm 8.76$ years. Ten patients with migraine with aura (group B) (nine females (90\%) and 1 male (10\%)) had a mean age of 27.50 \pm 7.08 years. There was no statistically significant difference between both groups as regards age and gender.

\section{Changes of clinical data after TMS treatment}

Comparison between pre- and post-rTMS reveals a highly significant reduction in the number of headache attacks, duration of individual attack, number of pills per month, pain intensity, MIDAS score, and MIDAS grade post-TMS in comparison with pre-TMS $(P$ value < 0.001 ) in the patient group (Table 1).

Generally, treatment of migraine by rTMS was easy to be applied and well tolerated by the patients. No facial twitching, headache, mood changes, tinnitus, dizziness, fatigue, or more serious adverse effects (e.g., seizures) were reported.

\section{Results of serum neurokinin A level}

There was a significantly higher serum level of neurokinin $\mathrm{A}$ in patients with migraine than in the control group $(P$ value $<0.001)$ (Table 2$)$.

After TMS, there was a highly significant reduction in the serum level of neurokinin A in the patient group (Table 2). However, still serum level in the patient group was significantly higher than that in the control group (Table 2).

Comparison between the pre- and post-rTMS reveals a highly significant reduction in the number of headache attacks, duration of individual attack, number of pills per month, pain intensity, MIDAS score, and serum level of neurokinin A in group A (migraine without aura) (Table 3).

Also, comparison between the pre- and post-rTMS reveals a highly significant reduction in the number of headache attacks, duration of individual attack, number of pills per month, pain intensity, MIDAS score, and serum level of neurokinin A in group B (migraine with aura) (Table 4).

There was a positive correlation between neurokinin A serum level and number of headache attacks, duration of individual attack, number of pills per month, pain intensity, and MIDAS score pre- and post-TMS (Tables 5 and 6; Figs. 1 and 2).

\section{Discussion}

In this study, the tested rTMS protocol was effective in the overall reduction in days of migraine/month, duration of migraine attack, number of analgesics taken during acute attacks, intensity of pain, and degree of disability assessed by MIDAS score 4 weeks after the sessions. In addition, we noticed that improvement even has lasted more than 1 month in five patients who were followed up to 6 months considering decreasing the 
Table 1 Comparison between 4 weeks pre- and 4 weeks post-TMS treatment as regards clinical data of migraine and MIDAS in the patient group

\begin{tabular}{|c|c|c|c|c|c|}
\hline & & Pre-TMS, no. $=40$ & Post-TMS, no. $=40$ & Test value & $P$ value \\
\hline \multirow[t]{2}{*}{ No. of headache attacks (days per month) } & Median (IQR) & $17.00(16-22.5)$ & $5.00(2-8)$ & $-5.515^{\ddagger}$ & $<0.001$ \\
\hline & Range & $7-27$ & $0-25$ & & \\
\hline \multirow[t]{2}{*}{ Duration of individual attack (hours) } & Median (IQR) & $15.63(12.5-19.5)$ & $0.25(0.13-0.53)$ & $-5.484^{\ddagger}$ & $<0.001$ \\
\hline & Range & $4.75-42$ & $0-19.50$ & & \\
\hline \multirow[t]{2}{*}{ No. of pills (per month) } & Median (IQR) & $23.00(15-33)$ & $0.00(0-1)$ & $-5.345^{\ddagger}$ & $<0.001$ \\
\hline & Range & $0-79$ & $0-33$ & & \\
\hline \multirow[t]{2}{*}{ Pain intensity } & Median (IQR) & $7.50(7.25-7.75)$ & $1.63(0.5-2.25)$ & $-5.450^{\ddagger}$ & $<0.001$ \\
\hline & Range & $5.25-8.75$ & $0-7.75$ & & \\
\hline \multirow[t]{2}{*}{ MIDAS score } & Median (IQR) & $31.00(22.5-44)$ & $2.00(0-4.5)$ & $-5.492^{\ddagger}$ & $<0.001$ \\
\hline & Range & $6-57$ & $0-50$ & & \\
\hline \multirow[t]{4}{*}{ MIDAS grade } & । & $0(0.0 \%)$ & $33(82.5 \%)$ & $62.280^{*}$ & $<0.001$ \\
\hline & $\|$ & $3(7.5 \%)$ & $4(10.0 \%)$ & & \\
\hline & III & $5(12.5 \%)$ & $1(2.5 \%)$ & & \\
\hline & IV & 32 (80.0\%) & $2(5.0 \%)$ & & \\
\hline
\end{tabular}

$P$ value $>0.05$, non-significant (NS); $P$ value $<0.05$, significant $(\mathrm{S}) ; P$ value $<0.001$, highly significant (HS)

${ }^{*}$ Chi-square test

"Wilcoxon's rank test

effect of TMS with increasing the period post-sessions. Our results were partially consistent with those of Teepker et al. [12] who treated twenty-seven migraineurs with lowfrequency rTMS $(1 \mathrm{~Hz})$ using the same protocol with a round coil; the study was a placebo-controlled, blinded study that resulted in a moderate and significant reduction regarding migraine attacks, days with migraine, and total hours with migraine, whereas no effects were evident for pain intensity and the use of analgesics.

This improvement in these migraine parameters can be explained by reduction in cortical excitability caused by low-frequency rTMS. Central neuronal hyperexcitability is proposed to be the putative basis for the physiological disturbances in migraine. In addition, studies of the visual cortex in patients with migraine have generally concluded that migraine (particularly migraine with aura) is associated with a state of functional cortical hyperexcitability [2].

In another explanation regarding the effects of TMS in migraine, previous studies have also suggested that blood flow and metabolic changes at the stimulation site, brain-derived neurotrophic factor upregulation, improvements in synaptic plasticity, and changes in the activity of the neural circuitry of the dorsolateral prefrontal (DLPF C)-cingulate cortex, including both the DLPFC and the anterior cingulate cortex, are involved [13].

Other studies, moreover, have demonstrated that the suppressive effects of low rTMS can be propagated to other regions not directly stimulated, presumably by functional connections. For instance, low rTMS of the left primary motor cortex reduces motor evoked potentials elicited by single-pulse TMS administered to the

Table 2 Comparison between the patient group (pre-TMS) and control group as regards neurokinin A serum level, and comparison between pre-TMS and post-TMS treatment neurokinin A serum level in the patient group

\begin{tabular}{|c|c|c|c|c|c|}
\hline Neurokinin A serum level & & Control group, no. $=20$ & Patient group, no. $=40$ & Test value ${ }^{\circ}$ & $P$ value \\
\hline \multirow[t]{2}{*}{ Pre-TMS } & Mean \pm SD & $1.15 \pm 0.28^{*}$ & $24.11 \pm 2.26$ & -45.131 & $<0.001$ \\
\hline & Range & $0.51-1.85$ & $19.38-27.93$ & & \\
\hline \multirow[t]{2}{*}{ Post-TMS in patient group } & Mean \pm SD & $1.15 \pm 0.28^{*}$ & $12.60 \pm 3.08$ & -16.524 & $<0.001$ \\
\hline & Range & $0.51-1.85$ & $9.32-25.45$ & & \\
\hline Paired $t$ test & & & -5.511 & & \\
\hline$P$ value & & & $<0.001$ (HS) & & \\
\hline
\end{tabular}

$P$ value $>0.05$, non-significant (NS); $P$ value $<0.05$, significant $(\mathrm{S}) ; P$ value $<0.01$, highly significant $(\mathrm{HS})$

- Independent $t$ test

*Neurokinin A serum level was calculated in the control group one time used in the comparison with the patient group serum level pre-TMS and post-TMS 
Table 3 Comparison between 4 weeks pre- and 4 weeks post-TMS as regards clinical data of migraine, MIDAS, and serum levels of neurokinin A in group A (migraine without aura)

\begin{tabular}{|c|c|c|c|c|c|}
\hline & & \multicolumn{2}{|l|}{ Without aura } & \multirow{2}{*}{$\begin{array}{l}\text { Test } \\
\text { value }\end{array}$} & \multirow[t]{2}{*}{$P$ value } \\
\hline & & Pre-TMS, no. $=30$ & Post-TMS, no. $=30$ & & \\
\hline \multirow[t]{2}{*}{ Neurokinin A serum level } & Mean \pm SD & $24.26 \pm 2.38$ & $12.44 \pm 2.94$ & \multirow[t]{2}{*}{$-4.782^{\circ}$} & \multirow[t]{2}{*}{$<0.001$} \\
\hline & Range & $19.38-27.93$ & $9.32-25.45$ & & \\
\hline \multirow{2}{*}{$\begin{array}{l}\text { No. of headache attacks } \\
\text { (days per month) }\end{array}$} & Median (IQR) & $17.50(16.00-25.00)$ & $6.00(2.00-8.00)$ & \multirow[t]{2}{*}{$-4.786^{\ddagger}$} & \multirow[t]{2}{*}{$<0.001$} \\
\hline & Range & $7.00-27.00$ & $0.00-25.00$ & & \\
\hline \multirow{2}{*}{$\begin{array}{l}\text { Duration of individual } \\
\text { attack (hours) }\end{array}$} & Median (IQR) & $15.63(12.75-18.25)$ & $.25(0.13-0.38)$ & \multirow[t]{2}{*}{$-4.762^{\ddagger}$} & \multirow[t]{2}{*}{$<0.001$} \\
\hline & Range & $4.75-42.00$ & $0.00-19.50$ & & \\
\hline \multirow[t]{2}{*}{ No. of pills (per month) } & Median (IQR) & $27.00(15.00-33.00)$ & $0.00(0.00-2.00)$ & \multirow[t]{2}{*}{$-4.683^{\ddagger}$} & \multirow[t]{2}{*}{$<0.001$} \\
\hline & Range & $0.00-79.00$ & $0.00-33.00$ & & \\
\hline \multirow[t]{2}{*}{ Pain intensity } & Median (IQR) & $7.50(7.25-7.75)$ & $1.75(0.75-2.50)$ & \multirow[t]{2}{*}{$-4.708^{\ddagger}$} & \multirow[t]{2}{*}{$<0.001$} \\
\hline & Range & $5.25-8.75$ & $0.00-7.75$ & & \\
\hline \multirow[t]{2}{*}{ MIDAS score } & Median (IQR) & $30.50(23.00-39.00)$ & $2.00(0.00-5.00)$ & \multirow[t]{2}{*}{$-4.783^{\ddagger}$} & \multirow[t]{2}{*}{$<0.001$} \\
\hline & Range & $6.00-57.00$ & $0.00-50.00$ & & \\
\hline
\end{tabular}

$P$ value $>0.05$, non-significant (NS); $P$ value $<0.05$, significant $(\mathrm{S}) ; P$ value $<0.001$, highly significant (HS)

'Paired $t$ test

"Wilcoxon's rank test

right primary motor cortex, an effect presumably mediated by transcallosal projections [14].

The reduction in pain intensity in our study and subsequently reduction in abortive analgesics intake but not in Teepker et al.'s study may be explained by the use of figureof-eight coil in our study while using round coil in Teepker et al.'s [12] study assuming that figure-of-eight coil is more effective than round coil as proven by Rollnik et al. [15].

In contrast to a circular coil, a figure-of-eight coil in which two coils are placed beside each other, wired such that the stimulator current rotates in opposite directions in the two coils, produces a more localized peak induced field and can decrease the uncertainty as to the site of stimulation. In fact, the area under the center of the side-by-side coils experiences approximately twice the induced electric fields that occur elsewhere in the vicinity of the coils [16].

These results are consistent with the study made by Tamura et al. who revealed that low-frequency rTMS over M1 induced earlier recovery from acute

Table 4 Comparison between 4 weeks pre- and 4 weeks post-TMS as regards clinical data of migraine, MIDAS, and serum levels of neurokinin A in group B (migraine with aura)

\begin{tabular}{|c|c|c|c|c|c|}
\hline & & \multicolumn{2}{|l|}{ With aura } & \multirow{2}{*}{$\begin{array}{l}\text { Test } \\
\text { value }\end{array}$} & \multirow[t]{2}{*}{$P$ value } \\
\hline & & Pre-TMS, no. $=10$ & Post-TMS, no. $=10$ & & \\
\hline \multirow[t]{2}{*}{ Neurokinin A serum level } & Mean \pm SD & $23.64 \pm 1.87$ & $13.09 \pm 3.58$ & $-2.803^{\circ}$ & 0.005 \\
\hline & Range & $20.46-26.45$ & $9.69-22.55$ & & \\
\hline \multirow{2}{*}{$\begin{array}{l}\text { No. of headache attacks } \\
\text { (days per month) }\end{array}$} & Median (IQR) & $16.50(15.00-20.00)$ & $3.00(2.00-6.00)$ & $-2.807^{\ddagger}$ & 0.005 \\
\hline & Range & $12.00-21.00$ & $0.00-20.00$ & & \\
\hline \multirow{2}{*}{$\begin{array}{l}\text { Duration of individual } \\
\text { attack (hours) }\end{array}$} & Median (IQR) & $15.00(12.25-25.50)$ & $.25(0.13-0.69)$ & $-2.803^{\ddagger}$ & 0.005 \\
\hline & Range & $4.75-28.50$ & $0.00-13.00$ & & \\
\hline \multirow[t]{2}{*}{ No. of pills (per month) } & Median (IQR) & $19.50(12.00-26.00)$ & $0.00(0.00-0.00)$ & $-2.666^{\ddagger}$ & 0.008 \\
\hline & Range & $0.00-36.00$ & $0.00-20.00$ & & \\
\hline \multirow[t]{2}{*}{ Pain intensity } & Median (IQR) & $7.50(7.25-7.75)$ & $.75(0.50-2.00)$ & $-2.812^{\ddagger}$ & 0.005 \\
\hline & Range & $6.50-8.50$ & $0.00-7.00$ & & \\
\hline \multirow[t]{2}{*}{ MIDAS score } & Median (IQR) & $35.00(22.00-46.00)$ & $2.00(0.00-4.00)$ & $-2.705^{\ddagger}$ & 0.007 \\
\hline & Range & $17.00-49.00$ & $0.00-47.00$ & & \\
\hline
\end{tabular}

$P$ value $>0.05$, non-significant (NS); $P$ value $<0.05$, significant $(\mathrm{S}) ; P$ value $<0.001$, highly significant $(\mathrm{HS})$

'Paired $t$ test

"Wilcoxon's rank test 
Table 5 Correlation between neurokinin A serum level and clinical parameters of migraine pre-TMS

\begin{tabular}{lll}
\hline & \multicolumn{2}{l}{ Neurokinin A serum level pre-TMS } \\
\cline { 2 - 3 } & $\boldsymbol{R}^{\perp}$ & $\boldsymbol{P}$ value \\
\hline No. of headache attacks (days per month) pre-TMS & $\mathbf{0 . 8 1 8}$ & $<\mathbf{0 . 0 0 1}$ \\
Duration of individual attack (hours) pre-TMS & $\mathbf{0 . 4 2 4}$ & $\mathbf{0 . 0 0 6}$ \\
No. of pills (per month) pre-TMS & 0.389 & 0.013 \\
Pain intensity (on scale from 0 to 10) pre-TMS & 0.421 & 0.007 \\
MIDAS score pre-TMS & 0.329 & 0.038 \\
\hline
\end{tabular}

$P$ value $>0.05$, non-significant (NS); $P$ value $<0.05$, significant $(S) ; P$ value $<0.01$, highly significant $(H S)$

$\stackrel{\perp}{\perp}$ Pearson's correlation coefficient test

pain compared with the sham or control conditions after induction of acute pain by intradermal capsaicin injection. This modulation of pain is thought to be through modification of $\mathrm{C}$-fiber pathways by slow rTMS [17].

The current study revealed non-significant difference between patients with migraine without aura and migraine with aura regarding the response to rTMS sessions. However, this may be due to the small number of the patients with migraine with aura in our study. This corresponds to the conclusion of Misra et al.'s study which cited that there is no significant difference between patients with migraine with aura and patients with migraine without aura regarding to the response to rTMS [18]. This also can be explained through that most of the neurophysiological characteristics are certainly common to migraine patients with and without aura because most patients with migraine with aura also experience migraine attacks without aura [19].

Neurokinin A plays an important role in the pathophysiology of migraine through enhancing the neurovascular inflammation mechanism being released from primary sensory neurons of the trigeminal ganglia (TG) and increasing its level during the migraine attack [20].

In this study, there was a highly significant increase in neurokinin A serum level in the patient group in comparison to the control group before rTMS sessions. However, after sessions, there was a reduction in neurokinin
A serum level by about $50 \%$ in the patient group. Nevertheless, it is still higher than the control group. This may be due to the neurovascular inflammatory processes, which have long been implicated as a possible mechanism in the pathophysiology of migraine. The results also revealed a positive correlation between neurokinin A serum level and number of headache attacks, duration of individual attack, number of pills per month, pain intensity, and MIDAS score pre- as well as post-TMS treatment. These results are in agreement with Sarchielli et al.'s [21] study results where ten migraineurs were treated with rizatriptan. He assessed neurokinin A serum level before and after treatment, where he found a significant decrease in neurokinin A serum level with significant pain relief and alleviation of accompanying symptoms. Patients who did not respond to rizatriptan had less significant variations in neurokinin A serum level before and following rizatrip$\tan$ administration, which indicate that neurokinin $\mathrm{A}$ is a good indicator for migraine improvement.

Generally, treatment of migraine by rTMS was easy to be applied and well tolerated by the patients, with minimal (e.g., mild dysesthesias and scalp discomfort) or no side effects.

Limitations of the study were the relatively small sample of migraine patients and no control group for TMS as the sham group. In addition, low-frequency TMS was used, while most studies used high-frequency TMS.

Table 6 Correlation between neurokinin A serum level and clinical parameters of migraine post-TMS

\begin{tabular}{lll}
\hline & \multicolumn{2}{l}{ Neurokinin A serum level post-TMS } \\
\cline { 2 - 3 } & $\boldsymbol{R}^{\perp}$ & $\boldsymbol{P}$ value \\
\hline No. of headache attacks (days per month) post-TMS & $\mathbf{0 . 8 2 4}$ & $<\mathbf{0 . 0 0 1}$ \\
Duration of individual attack (hours) post-TMS & $\mathbf{0 . 8 2 0}$ & $<\mathbf{0 . 0 0 1}$ \\
No. of pills (per month) post-TMS & $\mathbf{0 . 7 7 4}$ & $<\mathbf{0 . 0 0 1}$ \\
Pain intensity (on scale from 0 to 10) post-TMS & $\mathbf{0 . 8 2 7}$ & $<\mathbf{0 . 0 0 1}$ \\
MIDAS score post-TMS & $\mathbf{0 . 8 5 4}$ & $<\mathbf{0 . 0 0 1}$ \\
\hline
\end{tabular}

$P$ value $>0.05$, non-significant (NS); $P$ value $<0.05$, significant $(S) ; P$ value $<0.01$, highly significant (HS)

${ }^{\perp}$ Pearson's correlation coefficient test 


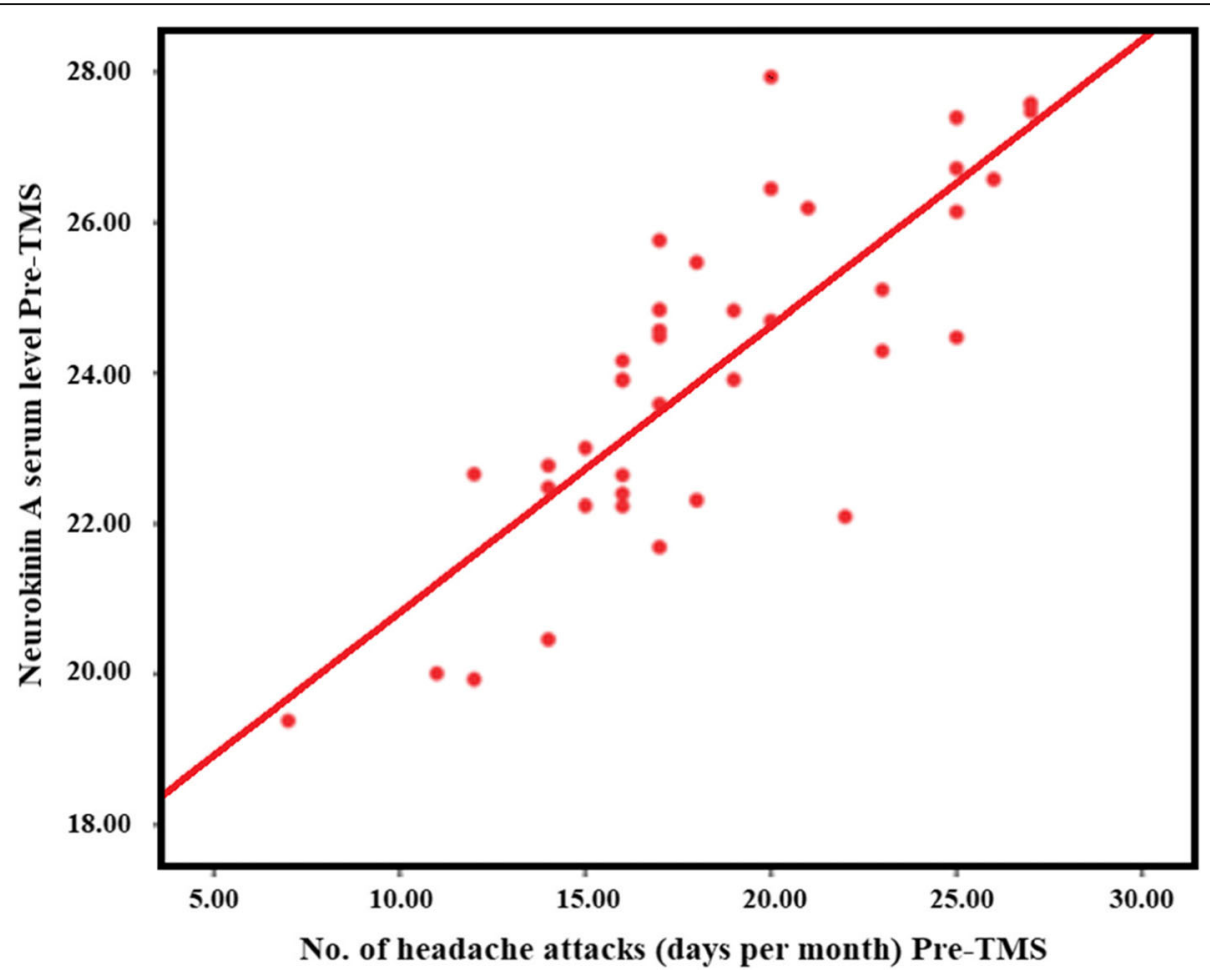

Fig. 1 Correlation between neurokinin A serum level and number of headache attacks pre-TMS

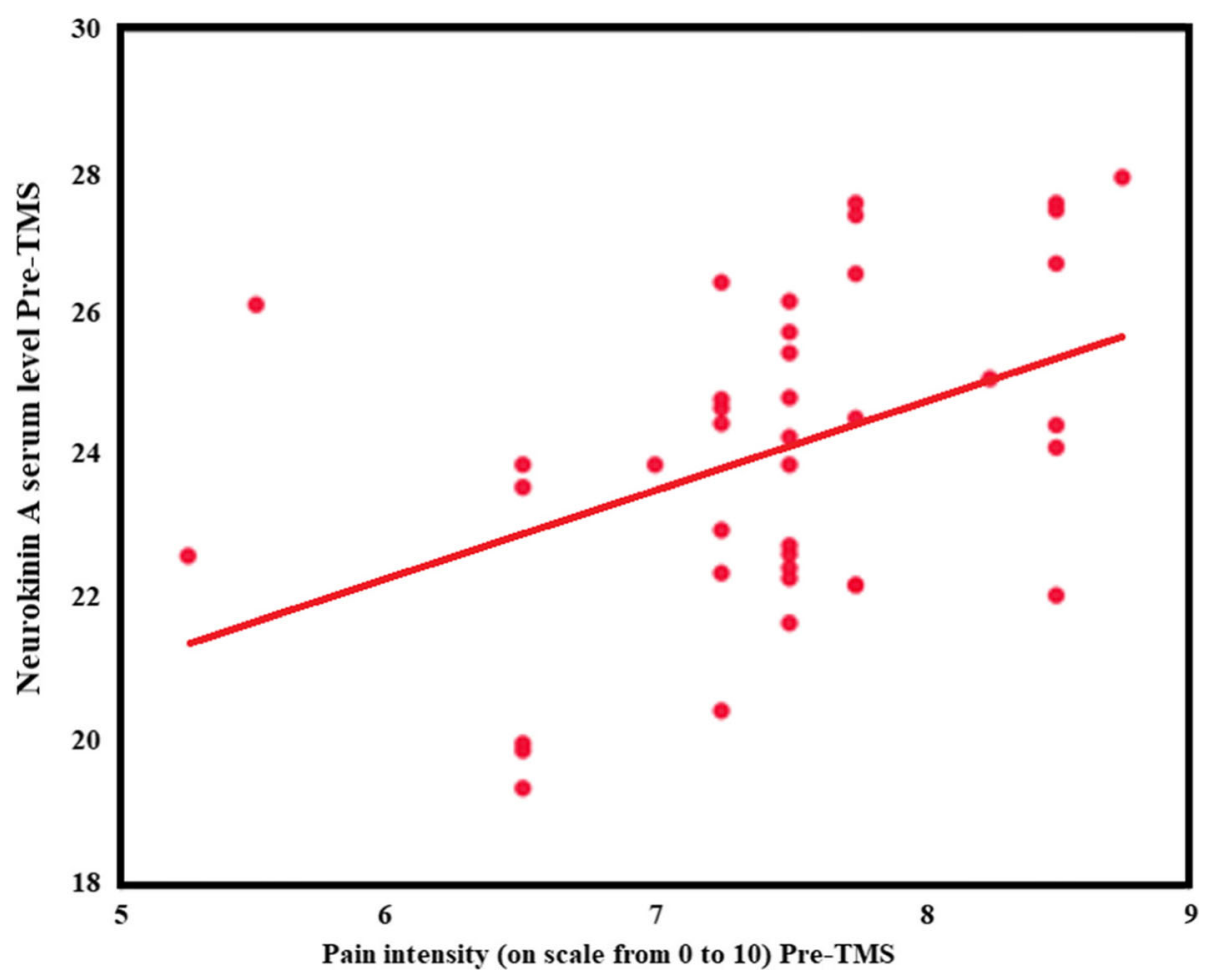

Fig. 2 Correlation between neurokinin A serum level and pain intensity pre-TMS 


\section{Conclusion}

In conclusion, the tested low-frequency rTMS protocol was easy to be applied, well-tolerated, safe, and effective method for migraine prevention with less or no side effects. Further research examining non-daily treatment rTMS protocol among larger study populations with variable migraine subtypes is warranted.

\section{Abbreviations}

BDHD: Basic Diagnostic Headache Diary; CGRP: Calcitonin gene-related peptide; CSD: Cortical spreading depression; EEG: Electroencephalogram; EIA: Enzyme Immunoassay; MIDAS: Migraine Disability Assessment scale; NO: Nitric oxide; rTMS: Repetitive transcranial magnetic stimulation; SPSS: Statistical Program for Social Science; TG: Trigeminal ganglia; TMS: Transcranial magnetic stimulation; 5-HT: 5-Hydroxytryptamine

\section{Acknowledgements}

The authors acknowledge the subjects for their participation and cooperation in this study.

\section{Authors' contribution}

$A B$ and RE contributed to the research idea, data analysis, interpretation, and manuscript review. GS contributed to the data acquisition, data analysis, interpretation, and manuscript writing and review. All authors read and approved the final manuscript.

\section{Funding}

The authors did not receive any funding for this work.

\section{Availability of data and materials}

The datasets used during the current study are available from the corresponding author on reasonable request.

\section{Ethics approval and consent to participate}

The Ethical Committee of the Faculty of Medicine of Al Azhar University approved the study. The number is not available. An informed written consent was taken from each patient involved in this study prior to the conduct of any study-related activities. All data obtained from every patient were confidential and were not used outside the study.

\section{Consent for publication}

Not applicable

\section{Competing interests}

None of the authors has any conflict of interest.

Received: 22 June 2020 Accepted: 23 November 2020

Published online: 07 January 2021

\section{References}

1. Straube A, Andreou A. Primary headaches during lifespan. The Journal of Headache and Pain. 2019;20:35.

2. Goadsby P, Holland P, Martins-Oliveira M, Hoffmann J, Schankin C, Akerman S. Pathophysiology of migraine: a disorder of sensory processing. Physiol Rev. 2017:97(2):553.

3. Goadsby P. Pathophysiology of migraine. Ann Indian Acad Neur. 2012; 15(Suppl. 1):S15-22.

4. Zardouz S, Shi L, Leung A. A feasible repetitive transcranial magnetic stimulation clinical protocol in migraine prevention. SAGE Open Med Case Rep. 2016:4:1-5.

5. Stilling JM, Monchi O, Amoozenger F, Debert CT. Transcranial magnetic and direct current stimulation(TMS / tDCS) for the treatment of headache: a systematic review. Headache. 2019;59(3):339-57.

6. Lipton R, Pearlman S. Transcranial magnetic simulation in the treatment of migraine. Neurotherapeutics. 2010;7(2):204-12.

7. Almaraz A, Dilli E, Dodick D. The effect of prophylactic medications on TMS for migraine aura. Headache. 2010;50(10):1630-3.

8. Hulla R, Liegey-Dougall A. A systematic review of high-frequency transcranial magnetic stimulation on motor cortex areas as a migraine preventive treatment. Cephalalgia Rep. 2019;2:1-13.
9. Ramachandran R. Neurogenic inflammation and its role in migraine. Semin immunopathol. 2018:40(3):301-14.

10. Headache Classification Committee of the International Headache Society (IHS). The International Classification of Headache Disorders, 3rd edition. Cephalalgia. 2018;38(1):1-211.

11. Jensen R, Tassorelli C, Rossi P, Allena M, Osipova V, Steiner T, et al. A basic diagnostic headache diary (BDHD) is well accepted and useful in the diagnosis of headache. A multicentre European and Latin American study. Cephalalgia. 2011;31(15):1549-60.

12. Teepker M, Hötzel J, Timmesfeld N, Reis J, Mylius V, Haag A, et al. Lowfrequency rTMS of the vertex in the prophylactic treatment of migraine. Cephalalgia. 2010;30(2):137-44.

13. Leahu P, Matei A, Groppa S. Transcranial magnetic stimulation in migraine prophylaxis. Journal of Medicine and Life. 2018;11(2):175-6.

14. Schambra H. Repetitive transcranial magnetic stimulation for upper extremity motor recovery: does it help? Curr Neurol Neurosci Rep. 2018; 18(12):97.

15. Rollnik J, Wüstefeld S, Däuper J, Karst M, Fink M, Kossev A, et al. Repetitive transcranial magnetic stimulation for the treatment of chronic pain - a pilot study. Eur Neurol. 2002;48(1):6-10.

16. Barker A, Shields K. Transcranial magnetic stimulation: basic principles and clinical applications in migraine. Headache. 2017:57(3):517-24.

17. Tamura Y, Okabe S, Ohnishi T. N Saito D, Arai N, Mochio S, et al.: Effects of $1-\mathrm{Hz}$ repetitive transcranial magnetic stimulation on acute pain induced by capsaicin. Pain. 2004;107(1-2):107-15.

18. Misra U, Kalita J, Bhoi S. High-rate repetitive transcranial magnetic stimulation in migraine prophylaxis: a randomized, placebo-controlled study. J Neurol. 2013;260(11):2793-801.

19. Charles A. The pathophysiology of migraine: ilmplications for clinical management. Lancet Neurol. 2018;17(2):174-82.

20. Buture A, Gooriah R, Nimeri R, Ahmed F. Current understanding on pain mechanism in migraine and cluster headache. Anesthesiol Pain Med. 2016; 6(3):e35190.

21. Sarchielli P, Pini L, Zanchin G, Alberti A, Maggioni F, Rossi C, et al. Clinical-biochemical correlates of migraine attacks in rizatriptan responders and non- responders. Cephalalgia. 2006;26(3):257-65.

\section{Publisher's Note}

Springer Nature remains neutral with regard to jurisdictional claims in published maps and institutional affiliations.

\section{Submit your manuscript to a SpringerOpen ${ }^{\circ}$ journal and benefit from:}

- Convenient online submission

- Rigorous peer review

- Open access: articles freely available online

- High visibility within the field

- Retaining the copyright to your article

Submit your next manuscript at $>$ springeropen.com 